\title{
ANÁLISIS Y PROSPECTIVA DEL DESARROLLO TURÍSTICO EN LOS PARQUES NATURALES DEL NORTE DE LA COMUNITAT VALENCIANA
}

\author{
Ouafae Abdelouahab Reddam \\ Diego López Olivares \\ dlopez@uji.es \\ Juan Bautista Ferreres Bonfill \\ Universitat Jaume I
}

\section{RESUMEN}

Las nuevas tendencias de la demanda turística en las últimas décadas han supuesto un creciente atractivo de nuevas tipologías turísticas entre ellas el turismo en los parques naturales. Los nuevos usos de los parques deben representar beneficios para la población local pero esos procesos deben ir acompañados de planificaciones de carácter sostenible. Para ello hemos aplicado en esta investigación una metodología Delphi a los parques naturales del interior-norte de la Comunitat Valenciana: Parque Natural de la Sierra de Espadán, Parque Natural del Penyagolosa y Parque Natural de la Tinença de Benifassà.

Palabras clave: Planificación estratégica integral, método Delphi, dinamización, sostenibilidad.

\section{ABSTRACT}

The new trends in tourist demand in the last few decades have involved the increasing attraction of new types of tourism, including tourism in natural parks. The new uses of the parks should lead to benefits for the local population, but those processes must be accompanied by sustainable planning. For this purpose, in this study we have applied Delphi methodology to the inland natural parks of the northern Valencian region: Sierra de Espadán Natural Park, Penyagolosa Natural Park and the Tinença de Benifassà Natural Park.

Keywords: Integrated strategic planning, Delphi method, dynamization, sustainability.

Fecha de recepción: marzo 2016.

Fecha de aceptación: febrero 2017. 


\section{LOS PARQUES NATURALES DEL INTERIOR NORTE DE LA COMUNITAT VALENCIANA: PN SIERRA DE ESPADÁN, PN PENYAGOLOSA Y PN TINENÇA DE BENIFASSÀ}

\section{I.1. Introducción}

Desde los años setenta del siglo pasado el turismo se ha convertido en una actividad económica que representa el 9\% del PIB mundial, y genera uno de cada 11 puestos de trabajo en el mundo. Es un sector fundamental de generación de ingresos en las economías emergentes y en desarrollo. Según los datos de OMT, de septiembre de 2014, presenta incrementos interanuales de demanda en los últimos lustros del 3,4\%; y en lo que respecta a la demanda en los parques naturales, esta oscila entre el 10 y el $30 \%$ de crecimiento interanual (López, 2014).

Estos cambios en la demanda turística condicionan la creciente competencia en el marco de una sociedad post industrial de una demanda turística con comportamientos más individualizados, por lo tanto menos gregarios, a la vez que su experiencia la ha convertido en más exigente con los productos. Exigencia no solamente en la relación calidad precio, sino en términos integrales de la oferta en el destino, donde pasan a tener un papel básico los aspectos relacionados con la naturaleza y la cultura (López et al.2005). Ello representa un creciente atractivo de nuevas tipologías turísticas entre ellas el turismo en los parques naturales (Marchena, 1992; Weaver, 2001; Epler, 2002; Buckley, 2003; Fenell y Dowling, 2003; Pulido, 2005; Newsone et al, 2005).

Ello viene acompañado por los avances tecnológicos que están facilitando el acceso a lugares a través de internet, proporcionando información actualizada sobre los lugares que el turista desea visitar (Huéscar, 2007; López y Pulido, 2007).

En este nuevo contexto, el medio ambiente es una de las preocupaciones de los visitantes, ya que un gran numero manifiesta la necesidad de que el turismo no tenga un gran impacto sobre el medio ambiente, por lo tanto a la calidad del producto o de destino turístico hay que añadir la calidad ambiental, si queremos conseguir la satisfacción del cliente (Pulido, 2005).

Los nuevos usos de los parques naturales, tienen beneficios sobre la población (López, 2010; López, 2014) que reside dentro del área protegida, ya que estos nuevos usos generan empleo, crean nuevas empresas turísticas y por lo tanto diversifican la economía local (Capdepón, 2013 y 2015), aunque no en los términos que se planteaban hace unas décadas, sobre todo si no hay planificaciones adecuadas. Además un desarrollo mal planificado en los espacios protegidos puede tener consecuencias negativas para la población y los propios parques en términos de impactos sobre la vegetación, flora y fauna por la contaminación acústica, del aire, por emisiones de $\mathrm{CO}_{2}$, acuíferos, etc. como acumulación de las basuras, ruidos, entre otros. Por lo tanto ello nos obliga a aplicar modelos de desarrollo turístico en los parques naturales, adecuados a las características físico geográficas, naturales-paisajísticas, medio ambientales, socio económicas y culturales, que nos permitan alcanzar ese desarrollo equilibrado y sostenible para no caer en la llamada "mercantilización de la naturaleza" (Vera, et al, 1997 y 2011) en esa búsqueda de la calidad de vida de la sociedad post moderna.

La nueva situación del turismo en los espacios naturales debe responder a las nuevas exigencias medio ambientales, de los valores territoriales y de la política turística en base a las tendencias de los mercados (Anton et al. 2008). Así pues, estamos ante una necesaria 
reestructuración productiva y territorial que se debe mostrar como un instrumento útil para la unificación de diagnósticos, concretando acciones públicas y privadas, estableciendo a su vez un marco coherente de movilización y de cooperación entre los agentes sociales en el ámbito de la nueva gobernanza. Para ello es necesario apostar por la planificación estratégica e integral de la actividad turística con un enfoque sostenible pero además participativo.

Con estos planteamientos previos, hemos realizado el presente trabajo de investigación aplicando la técnica Delphi al análisis de partida y al estudio prospectivo de los parques naturales del interior- norte de la Comunitat Valenciana: El Parque Natural de la Sierra de Espadán, el Parque Natural del Penyagolosa y el Parque Natural de la Tinença de Benifassà.

\section{I.2. Características de los parques naturales de la Comunitat Valenciana: Los parques del inte- rior norte de la Comunitat}

Con 2.325.500 ha, la Comunitat Valenciana goza de una gran variedad de paisajes adornados por ríos, gargantas, y montañas acompañados de un marcado clima mediterráneo que ayuda a generar una diversidad singular y de gran importancia. Todo esto se puede valorar claramente en sus 22 parques naturales que alcanzan una superficie de 169.519,86 ha, repartidas entre las provincias de Alicante, Valencia y Castellón.

Así, bajo un contexto territorial y atendiendo a los valores del medio físico-natural, que singularizan estos espacios naturales protegidos a través de una diversidad fisiográfica, se pueden agrupar en sierras de interior, sierras litorales, humedales ya sean marjales o salinas, ríos e islas. Las sierras de interior ofrecen la mayor representatividad territorial, acogiendo 9 parques naturales (Chera-Sot de Chera; Desert de les Palmes; La Font Roja; Penyagolosa; Puebla de San Miguel; Serra de Mariola; Sierra de Espadán; Sierra Calderona; y La Tinença de Benifassà), cuya superficie protegida contrasta entre las 2.298 ha del parque natural de la Font Roja a las 31.180 ha del parque natural de la Sierra de Espadán. Las sierras litorales, aunque muy tensionadas por la presión urbanística, en algunos tramos del litoral se ha salvaguardado su valor paisajístico, lo cual ha posibilitado la declaración de los parques naturales de El Montgó; el Penyal d'Ifac; la Serra d’Irta y la Serra Gelada. Su extensión superficial es más modesta, protegiendo un total de 16.551,4 ha., que contrastan entre las 7.802,2 ha. de la Serra d'Irta, y las 53,3 ha. del parque natural del Penyal d'Ifac. Los humedales, que se corresponden con formaciones de marjales y salinas, considerando la superficie protegida, 31.659,4 ha., integran los parques naturales del Fondó d'Elx, L'Albufera, la Marjal de PegoOliva, el Prat de Cabanes-Torreblanca, las Lagunas de La Mata-Torrevieja, y las Salinas de Santa Pola. Entre ellos sobresale el parque natural de L'Albufera de Valencia con 20.960,7 ha. Los ríos además de su importancia ecológica y paisajística, son elementos vertebradores del territorio y favorecen el uso recreativo. En la Comunitat Valenciana existen dos parques naturales de estas características, ambos en la provincia de Valencia, el parque natural del Turia (4.736,3 ha.) y el de las Hoces de Cabriel que representa la mayor superficie protegida valenciana, alcanzando las 31.469,68 ha, situado en la comarca de Utiel-Requena. Por último, el parque natural de Les Illes Columbretes ubicado a 30 millas al este del Cap de Oropesa del Mar de Castellón, presenta un espacio protegido de 19 ha en tierra y 2.500 ha en el mar. 
El área de estudio de la presente investigación se concretará en: El parque natural de la Sierra de Espadán; el parque natural del Penyagolosa; y el parque natural de la Tinença de Benifassà.

Figura 1

MAPA UBICACIÓN Y DELIMITACIÓN DE LOS PARQUES NATURALES

DEL INTERIOR-NORTE DE LA COMUNITAT VALENCIANA

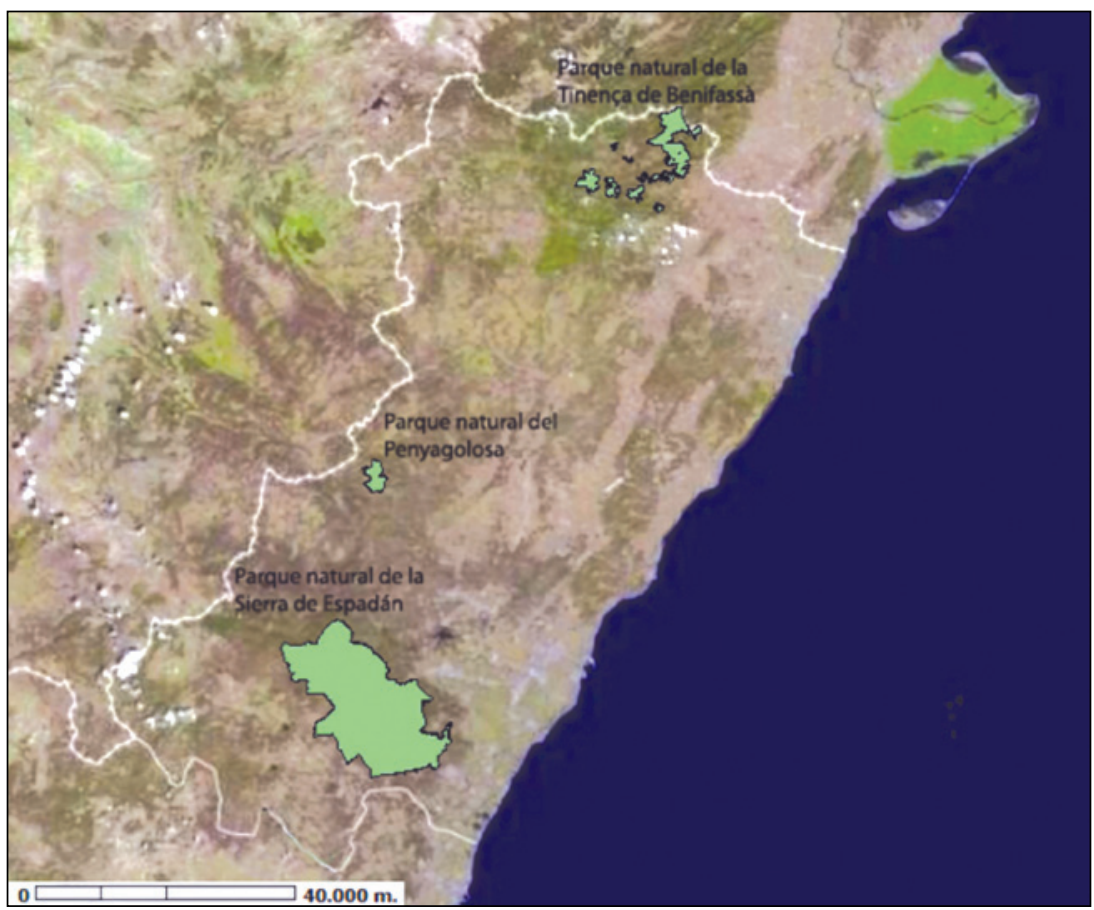

Fuente: Consellería de Agricultura, Medioambiente, Cambio Climático y Desarrollo Rural y elaboración propia.

\section{I.2.1. El Parque Natural de la Sierra de Espadán}

El Parque Natural de la Sierra de Espadán, fue declarado el 29 de septiembre de 1998 con el decreto 161/1998, de 29 de septiembre por el gobierno valenciano (DOGV 334708/10/1998), se localiza al sur de la provincia de Castellón entre las cuencas de los ríos Mijares y Palancia. Con una superficie de 31.180 ha, se considera el parque más importante y más extenso de la provincia de Castellón y el segundo de la Comunitat Valenciana.

La superficie protegida abarca 19 municipios con una población total de 7.898 habitantes pertenecientes a distintas comarcas, los cuales comprenden 4 categorías de uso: las áreas de protección ecológica representan el 85\% de la superficie, las áreas de uso agrícola el 12\%, las áreas de reserva con $2 \%$ y las áreas urbanas con $1 \%$.

Este parque natural presenta un alto valor geomorfológico, ecológico y paisajístico. En base al Plan de Ordenación de los Recursos Naturales (PORN) en su Zona de Protección 
(ZP) y áreas de Predominio Forestal (PF), destacan las especies forestales como los alcornoques (Quercussuber), las dos especies de pino dominantes el pino carrasco (Pinushalepensis) y pino rodeno (Pinuspinaster), las formaciones boscosas constituidas por la carrasca (Quercusrotundifolia), las formaciones de rebollo (Quercus pirenaica) localizadas en las laderas norte del Pico de la Rápita, las formaciones de quejigo (Quercusfaginea) localizadas en las laderas del Pico de la Rápita, Pico Espadán; también núcleos de tejos (TaxusBaccata) y acebos (Ilexaquifolia) entre otras, tenemos también las formaciones de matorral.

El Parque Natural de la Sierra de Espadán, en su Zona de Amortiguación de Impactos (ZAI), que incluye todas las masas forestales como arbolado, matorrales, presenta también un alto valor con una flora de gran interés ecológico, paisajístico y económico caso de la explotación de los alcornocales para la producción de corcho. Entre los valores paisajísticos destacamos por sus aportaciones medioambientales, agrícolas, sociales y turísticas las unidades paisajísticas formadas por las ramblas de Algímia- Azuébar y por las laderas de valles cultivados de Villamalur-Alcudia de Veo-Aín- Chóvar.

En el ámbito el Plan de Ordenación de Recursos Naturales (PORN) su Plan de Uso Público define dos tipos de áreas de uso recreativo: Uso Recreativo-Naturalístico y Uso Recreativo-Extensivo, como puede ser la utilización con fines didácticos o recreativos de las áreas. El PORN insta sobre la adecuación de las actividades que quedan sujetas a la conformidad con las normas particulares del PORN. También poner en valor los recursos turísticos de la Sierra de Espadán y dotarlos de la necesaria infraestructura de acompañamiento con el fin de atraer a la demanda de turismo de naturaleza y cultural.

\section{I.2.2. El Parque Natural del Penyagolosa}

El macizo de Penyagolosa se ubica al oeste de provincia Castellón, entre los términos municipales de Vistabella del Maestrat, Xodos y Villahermosa del Río, extendiéndose el parque, además de los términos citados, sobre los de Lucena del Cid y Castillo de Villamalefa con una población total de 2.623 habitantes. .

Con una extensión de 1.094,45 ha engloba el citado macizo de Sant Joan de Penyagolosa que alcanza una altura $1.814 \mathrm{~m}$, considerado el segundo techo más alto de la Comunitat Valenciana, superado el Cerro Calderón. El macizo del Penyagolosa forma una mezcla de ambientes geológicos, biológicos y socioculturales, con un entorno de cerros, senderos y barrancos que atraen a los excursionistas.

El Penyagolosa cuenta con varias figuras de protección, fue ZEPA (Zona de Especial Protección para las Aves) declarado en mayo de 2000, en julio 2001 propuesto como LIC (Lugar de Importancia Comunitaria) y declarado Parque Natural el 7 de abril 2006 con el Decreto49/2006. Una protección que se puede considerar fruto de la variedad de su flora y fauna. Entre la diversidad de su fauna destaca la existencia de grandes rapaces como el águila real, el gato montés, el corzo entre otros. En cuanto a la flora, sobresale la presencia del roble valenciano, tejo y formaciones de melojo muy interesantes. El 16 de noviembre de 1998, la Orden de la Conselleria de Medio Ambiente, declaró el Barranc de la Pegunta como microreserva vegetal protegiendo una zona de unas 20 ha, con especies endémicas, amenazadas o raras. 
En la zona se encuentran varias singularidades como asentamientos íberos, calzadas romanas, acogiendo también celebraciones de romerías, siendo la más conocida la de "los peregrinos de Les Useres" (Els Pelegrins de les Useres) entre otras como las de Vistabella del Maestrat, Culla, Xodos y Puertomingalvo. A parte del arraigado excursionismo de este espacio en la última década han destacado o potenciado actividades deportivas con un gran atractivo para la demanda.

\section{I.2.3. El Parque Natural de la Tinença de Benifassà}

El Parque Natural de la Tinença de Benifassà tiene una superficie de 4.967,548 ha extendiéndose en el noroeste castellonense por los términos municipales de la Pobla de Benifassà, Coratxà, el Boixar, Fredes, Bell (Rossell), Vallibona y Castell de Cabres (1.540 habitantes). La Tinença de Benifassà forma parte del gran macizo de los Puertos de Beceite, situado entre Aragón, Cataluña y la Comunitat Valenciana.

Este parque se distingue por un relieve abrupto y una masa forestal bien conservada, con el predominio del carrascal valenciano. A ello ha contribuido favorablemente la baja densidad de población y el uso exclusivo dedicado a pastos o explotaciones forestales lo que tuvo como fruto una estructura simple de la flora del Parque Natural de Benifassà y como resultado una mayor diversidad de ambientes que ha permitido el desarrollo de comunidades vegetales muy interesantes.

$\mathrm{Su}$ flora está formada por especies endémicas que han fomentado la declaración de diversas microreservas, especies como Pinguiculadertosensis, Antirrhinumpertegasii, Biscutellafonqueri o la Arenaria conimbricensisviridis, que son la grasilla tortosina, la dragoncilla de roca, el anteojos, y la arenaria...También atrae la fauna, caso de la cabra montesa Capra pirenaica hispanica. Destacar que su diversidad geológica de la que está dotada el área ha contribuido en facilitar un ambiente adecuado para la conservación de estas especies y otras rapaces, este ecosistema singular y variado aporta un gran valor a la Tinença de Benifassà.

Aparte del valor faunístico y florístico de la zona, el Parque tiene un rico patrimonio histórico-cultural y arquitectónico-religioso destacando la Cartuja de Santa María de Benifassà, la cual se ve inmersa en un interesante mundo rural, con sus tradiciones y elementos construidos en piedra seca, como neveras, hornos de cal, casetas de pastor, entre otros.

\section{OBJETIVOS Y METODOLOGÍA}

\section{Il.1. Objetivos de la investigación}

El objetivo general de la investigación consiste en alcanzar las bases que nos permitan conseguir un adecuado desarrollo turístico de los parques naturales teniendo siempre como referencia de futuro la sostenibilidad de estos espacios. Al respecto, los objetivos específicos son los siguientes:

1. Conocer en qué medida un desarrollo adecuado y planificado puede dinamizar el desarrollo turístico de los espacios naturales protegidos. 
2. Conocer, como área de estudio, la situación del turismo en los espacios naturales protegidos del interior-norte de la Comunitat Valenciana desde la óptica de los diferentes agentes públicos y privados.

3. Determinar las tendencias y prospectiva del futuro turístico en los parques naturales del espacio de la investigación.

4. Indicar las acciones y medidas que puedan ayudar a mejorar la situación turística de estos espacios naturales protegidos.

Para alcanzar estos objetivos se ha considerado adecuada la aplicación de la técnica Delphi como metodología capaz de conseguir los fines propuestos.

\section{II.2. El método Delphi: su aportación a la planificación turística}

El método para analizar esos factores y variables ha sido el método Delphi, técnica muy aplicada en las investigaciones de carácter social, tanto para alcanzar conclusiones como para valorar desde el punto de vista predictivo y prospectivo, con entrevistas a expertos, la evolución de una temática concreta. Muchos han sido los autores que han aplicado esta técnica desde la perspectiva de evolución y desde la vertiente prospectiva entre ellos: Landeta, 1999; Linstone, y Turoff, 1975, 2002; Sancho, 2003; Garrod, y Fyall, 2005; Hsu, y Sandford, 2007; entre otros.

El método Delphi según Linstone y Turoff (1975:3) “es un método de estructuración de un instrumento de comunicación grupal efectivo a la hora de permitir a un grupo de individuos, como un todo, tratar un problema complejo". Este método pretende ser un método sistemático e iterativo (Landeta, 1999:31), que consiste en la selección de un grupo de expertos para recabar sus opiniones de manera anónima y también buscar el consenso, si es posible. Esta herramienta de análisis parte de la composición de diferentes cuestionarios que nos permitirán acceder a los niveles de convergencia y divergencia del conjunto de entrevistados sobre la temática planteada.

Pulido (2005:70) lo define como "una técnica prospectiva de obtención de información cualitativa o subjetiva, pero relativamente precisa en contextos de información imperfecta fruto de combinar el conocimiento y experiencia de expertos en diversos campos, de una forma que tiende hacia el consenso de opiniones sobre futuros efectos específicos cuantificando estadísticamente a su vez estas opiniones mediante medidas tales como: media, mediana y cuartiles"

Entre los objetivos básicos que pretende el método Delphi destacamos: realizar previsiones; detectar las tendencias de futuro del espacio y temáticas a tratar; definir escenarios de futuro alternativos y programar estrategias de desarrollo.

En la investigación turística, el método Delphi se empezó a aplicar en el mundo anglosajón. Así, Garrod y Fyall (2005:88) citan autores que han implementado esta técnica en investigaciones de carácter internacional Moutinho y Witt (1995) en torno a las probabilidades de eventualidades que se sucediesen en la actividad turística. Igualmente, este método se ha aplicado en referencia a los impactos ambientales propiciados por el desarrollo turístico, caso de los estudios de Green et al. (1990). En el ámbito de los mercados turísticos se ha aplicado en Sudáfrica, Kaynak; Bloom, y Leibold, (1994), en concreto sobre convenciones y 
congresos la han aplicado Weber y Ladkin (2003). También se ha implementado esta técnica en un área del suroeste australiano para valorar el desarrollo local (Northcote, Lee, Chok y Wegner, 2008) y alcanzar el grado de implicación y participación de agentes locales.

Por lo que respecta a España, la aplicación del método es relativamente reciente, aunque han proliferado sus aplicaciones en los últimos años sobre todo en el ámbito universitario, más concretamente en el proceso de realización de tesis doctorales, y con temáticas muy variadas en torno al desarrollo turístico. En el año 2001, Fabiola López trata la temática de la ventaja competitiva de las agencias de viaje; en 2005 Ángel Celaya, aplica el método para definir la estrategia del turismo en Córdoba; en la temática del turismo rural para conocer el estado de la Comunitat Valenciana, aplica la técnica Javier Solsona (2010 y 2012); en 2014 en la Universidad de Málaga aplica en su tesis doctoral el método Delphi Luzardo Padrón en torno a las potencialidades turísticas de los Puertos de Alta Gracia (Venezuela). También para implementar procesos de gobernanza se ha hecho uso de esta técnica por parte de Pulido M. en su tesis doctoral leída en la Universidad de Jaén (Pulido, y Pulido, 2016).

Por último y en torno a la temática de esta investigación sobre los Parques Naturales y aplicando esta técnica Delphi destacan las tesis doctorales de Juan Ignacio Pulido (2005) sobre la gestión de los parques naturales andaluces y la de David Flores (2008) también sobre estos mismos espacios pero desde la perspectiva de la competitividad turística.

Esto sólo es una muestra de la aplicación de este método Delphi que a pesar de ciertos inconvenientes son más las ventajas que aportan, de ahí nuestra aplicación.

\section{II.3. Planteamiento de la investigación en los parques naturales del interior-norte de la Comuni- tat Valenciana}

El método Delphi es una de las bases empíricas aplicadas en esta investigación, sobre los parques naturales del norte-interior de la Comunitat Valenciana. Estos son: El PN de la Sierra de Espadán, PN de la Tinença de Benifassà y el PN del Penyagolosa. La aplicación del método Delphi nos ha permitido conocer las potencialidades del sistema turístico de estos espacios naturales, su prospectiva y poder ayudar a plantear estrategias de desarrollo con un enfoque integral. El trabajo de campo para aplicar la técnica Delphi se realizó entre octubre de 2014 y febrero de 2015 . La intención fue por una parte alcanzar el conocimiento de los elementos que han fijado la actividad en la actualidad, y por otra alcanzar una visión prospectiva por medio de la proyección de los factores que puedan condicionar el futuro del desarrollo de los parques naturales del área de estudio en los próximos años. Para ello, en primer lugar se seleccionó el panel de expertos a la vez que se configuró el cuestionario en el marco de las diferentes rondas de perfeccionamiento y finalmente la metodología de tabulación y análisis de los resultados.

\section{II.3.1. La selección de expertos en el área de estudio}

Uno de los aspectos más relevantes y complejos en la aplicación de la técnica Delphi fue la selección de los agentes sociales que deberían formar el panel de expertos. Aunque se seleccionaron expertos externos de las áreas de estudio, se consideró básico que los participantes tuviesen una estrecha relación entre la actividad profesional, institucional o inves- 
tigadora con estos espacios. Al respecto se detectaron cuatro sectores básicos que deberían formar parte del panel de expertos: los representantes empresariales, los representantes de la administración pública, los técnicos y especialistas del medio natural, y los académicos e investigadores (Tabla 1).

Tabla 1

FICHA TÉCNICA DEL ESTUDIO DELPHI

\begin{tabular}{|c|c|c|}
\hline Tamaño de la muestra & \multicolumn{2}{|c|}{$\begin{array}{l}\text { Primera ronda: } 25 \text { Expertos } \\
\text { Segunda ronda: } 23\end{array}$} \\
\hline Fecha de trabajo empírico & \multicolumn{2}{|c|}{$\begin{array}{l}1^{\mathrm{a}} \text { ronda: Octubre-noviembre } 2014 \\
2^{\mathrm{a}} \text { ronda: Enero- febrero } 2015\end{array}$} \\
\hline \multirow[t]{2}{*}{ Tipo de estudio } & Delphi & \\
\hline & Participantes $\mathbf{1}^{\mathrm{a}}$ ronda & Participantes $2^{\mathrm{a}}$ ronda \\
\hline $\mathbf{N}^{\mathbf{o}}$ & 25 & 23 \\
\hline Representantes empresariales & 7 & 5 \\
\hline Representantes de la administración pública & 5 & 5 \\
\hline Técnicos y especialistas en el medio natural & 6 & 6 \\
\hline Académicos e investigadores & 7 & 7 \\
\hline
\end{tabular}

Fuente: elaboración propia.

Por lo que respecta al sector empresarial representando a la pequeña y mediana empresa se seleccionaron empresas de alojamiento en sus diferentes ofertas (hotel, casa rural, entre otros.), restauración, de ofertas complementarias y de artesanía, en total siete personas en mayoría dueños o gerentes de empresas.

De la administración pública fueron cinco las personas seleccionadas, básicamente al más alto nivel de responsabilidad de los parques naturales del área de estudio, desde alcaldes o alcaldesas, o responsables de la dinamización de los parques naturales del área de estudio.

En cuanto a técnicos o especialistas se eligieron a aquellos que tienen o ha tenido alguna relación con los parques objeto de estudio con perfiles formativos de ciencias ambientales, sociología y turismo sostenible, siendo un total de seis.

Por último, el bloque de expertos académicos e investigadores, fueron siete entre profesores e investigadores de diversas universidades. Estos con una amplia y reconocida trayectoria en el conocimiento e investigación en espacios naturales protegidos. Además aportaban algo muy importante, la transversalidad de sus perfiles en la investigación turística, caso de economistas, geógrafos, sociólogos y especialistas en ciencias ambientales.

En definitiva, se formó un panel de 25 expertos de reconocido prestigio y gran experiencia en investigaciones de los espacios naturales protegidos. Muestra que se encuentra entre el intervalo de fidelidad (7 y 30 panelistas) que defienden diferentes autores como Landeta (1999) o Garrod y Fyall (2004), entre otros. 


\section{II.3.2. Diseño del cuestionario y las rondas de perfeccionamiento}

La elaboración del cuestionario se realizó a partir del conocimiento de las numerosas investigaciones que se disponen sobre los parques naturales, de las fuentes estadísticas y publicaciones de las instituciones como el Instituto Nacional de Estadística (INE); el Ministerio de Agricultura, Alimentación y Medio Ambiente; Instituto de Estudios Turísticos (IET); Organización Mundial del Turismo (OMT); Agència Valenciana del Turisme (AVT); y Europarc España (2009). Las consultas a estas instituciones se han completado con otras investigaciones realizadas sobre la temática, caso de Pulido (2005 y 2007); Anton et al. (2008), López et al. (2010) y Muñoz, (2008). Además de la realización del análisis de los elementos del sistema turístico de los parques naturales objeto de esta investigación.

En la metodología aplicada del método Delphi se optó por un cuestionario cerrado sobre todo en la primera parte, en la referente al análisis de la situación y en la parte del estudio prospectivo por un enfoque semicerrado. Ello como consecuencia de que la temática sobre los parques está bastante investigada y más que alcanzar los factores nos interesaba como estos se estaban desarrollando en opinión del panel de expertos. En cualquier caso cada entrevistado al final de cada pregunta podría añadir aquella que considerara importante y no se había preguntado.

El cuestionario se estructuró para la participación de los expertos en diferentes rondas. La primera ronda constaba de 126 cuestiones agrupadas en 15 preguntas. Estas se dividieron en dos bloques, uno configurando la primera parte y dedicado al análisis de partida (preguntas 1 a 6), y otra dedicada a la prospectiva (preguntas 7 a 15), incluyendo las preguntas de carácter abierto. Las cuestiones se plantearon en la escala Likert 5 (1 totalmente en desacuerdo; 2 bastante en desacuerdo; 3 ni de acuerdo ni en desacuerdo; 4 bastante de acuerdo; 5 totalmente de acuerdo).

En el apartado de análisis de partida, bloque I, en las seis preguntas realizadas a los entrevistados (Tabla 2) se pretendía conocer qué opinaban sobre los recursos turísticos (esta pregunta la componían 11 ítems), sobre los factores socioeconómicos y medioambientales (8 ítems o subpreguntas), la oferta turística (17 ítems), y la demanda turística (10 ítems), infraestructuras y servicios (12 ítems) y finalmente, 17 subpreguntas en torno a la financiación y gestión. A este total de 75 ítems se añadieron 3 subpreguntas para la segunda ronda en base a las sugerencias de los entrevistados alcanzando un total de 78 ítems o subpreguntas para este bloque de análisis de partida.

En el bloque II, el de prospectiva (Tabla 2) se dividía en dos apartados, el primero con 3 preguntas cerradas sobre el futuro del desarrollo en torno a los factores que pueden representar un freno a la buena evolución turística en los parques naturales del interior-norte de la Comunitat Valenciana, acciones para activar el desarrollo turístico en estos espacios naturales y finalmente la valoración futura sobre su desarrollo turístico sostenible. Las subpreguntas fueron 51 a las que se añadieron las introducidas para la segunda ronda las de los panelistas que fueron 5, en definitiva un total de 56 ítems.

En el segundo apartado de este segundo bloque la componían 6 preguntas de carácter abierto, cuyo contenido se refería a las nueva tendencias y hábitos futuros de la demanda, a las dificultades del futuro desarrollo de los parques, a las medidas a aplicar para el desarrollo sostenible de estos espacios, a la financiación y gestión y a la identificación de los problemas 
relacionados con el uso turístico de los espacios naturales protegidos del interior-norte de la Comunitat Valenciana. A estas preguntas cabía la posibilidad de que los entrevistados pudieran añadir sugerencias, caso que no sucedió.

Los cuestionarios fueron remitidos para la primera ronda en octubre y contestados en noviembre de 2014. Su tabulación fue concluida en diciembre de 2014, completando el cuestionario para la segunda ronda.

Ya en una segunda ronda, esta estaba dedicada a la propuesta de consenso y en ella se ofrecía a los entrevistados la posibilidad de modificar su opinión a aquellos que presentaban en sus respuestas una desviación respecto a la media de la primera ronda, quedando fuera del rango intercuartílico. Para ello se les envió unas hojas en las que constaban 4 apartados en el encabezamiento de las mismos: en la primera columna aparece la media de los resultados generales; en la segunda la desviación típica; en la tercera la puntuación propia, es decir la que cada entrevistado había aportado y por último una cuarta columna en blanco para el que quisiera modificar su opinión inicial de la primera ronda o en su caso dejarla en blanco. Ello se completaba con la opinión de los panelistas sobre las 5 subpreguntas propuestas por ellos en torno a los factores que podían representar: un freno a la evolución positiva de los parques; sobre el intrusismo laboral; la banalización de los paisajes naturales y culturales; modelo de desarrollo; o pérdida de biodiversidad asociada al modelo de desarrollo humano.

Esta segunda ronda fue enviada a los panelistas el 20 de enero de 2015, recibiendo las contestaciones en el mes siguiente.

Tabla 2

ESTRUCTURA Y CONTENIDO DEL CUESTIONARIO

\begin{tabular}{|l|c|}
\hline Preguntas: Análisis de factores & Ítems \\
\hline Bloque I: Análisis de partida & 11 \\
1. Recursos turísticos & 8 \\
2. Factores socioeconómicos y medio ambientales & 17 \\
3. La oferta turística & 10 \\
4. La demanda turística & 12 \\
5. Las infraestructuras y servicios & 17 \\
\hline 6. La financiación y gestión & \\
\hline Bloque II: Prospectiva & 18 \\
\hline 7. Factores que pueden representar un freno a la buena evolución turística en los PN & 22 \\
9. Acciones para activar el desarrollo turístico en los PN & 11 \\
\hline El desarrollo turístico sostenible en los PN & 126 \\
\hline
\end{tabular}

Fuente: elaboración propia.

Alcanzados los resultados de la segunda ronda y contrastando que la desviación típica en las diferentes subpreguntas se había reducido y que no había grandes diferencias entre las contestaciones de los expertos, deduciéndose esto de los resultados del rango intercuartilico que presentaban cifras casi similares a la mediana, se valoró que sería difícil conseguir un consenso más elevado entre los panelistas de ahí que se desistiese pasar una tercera ronda. 
Por lo tanto, con esta segunda ronda se daba por concluido el proceso del método Delphi, procediéndose a la tabulación entre los meses de abril a junio del 2015.

\section{II.3.3. Tabulación de los resultados}

Respecto a la tabulación de los resultados, la información aportada por las diferentes rondas del cuestionario se codificó y se almacenó en un fichero del programa SPSS 22.0, programa escogido para realizar el análisis de las aportaciones del cuestionario.

Este proceso ha consistido en la tabulación de las variables que tenía el cuestionario para alcanzar los principales estadísticos descriptivos. Todo ello fue almacenado en ficheros de sintaxis SPSS, es decir, en el lenguaje de programación del programa.

En la tabulación se empleó el comando de sintaxis CTABLES, al igual que los necesarios para el fichero de datos o información.

\section{PRINCIPALES RESULTADOS OBTENIDOS EN LA INVESTIGACIÓN}

\section{III.1. Análisis de partida}

Entre los principales resultados que se han producido en la aplicación de la técnica Delphi en el bloque I (Tabla 2) en función del nivel de consenso obtenido tanto en la desviación típica como en la valoración media, destacamos:

\section{III.1.1. Recursos turísticos}

Los miembros del panel de expertos valoraron los recursos turísticos como muy importantes para el desarrollo de la actividad turística en los espacios naturales protegidos. Los recursos turísticos más valorados fueron los senderos, caminos y pistas forestales, al igual que los recursos paisajísticos y de observación. También son muy bien valorados el patrimonio histórico-monumental y etnológico, el paisaje tradicional de los núcleos de población, la flora y fauna. Los entrevistados según los valores de la mediana y de la moda, (con valor máximo 5) se mostraron casi por unanimidad como considerándolos como recursos básicos para el desarrollo de la actividad turística en los PN del Interior-Norte de la Comunitat Valenciana. Igualmente el valor del consenso se muestra en la desviación típica muy alto para estas primeras subpreguntas oscilando entre el 0,5 y el 0,7.

El resto de subpreguntas también quedaron muy bien valoradas oscilando la media entre 4,4 para la gastronomía y el 3,2 para las concavidades subterráneas. Entre ambas se destacan las fuentes y manantiales, ríos, folclore y la artesanía. Siendo la mediana de 4 y la desviación típica entre 0,8 y 1,1, es decir se refleja también un alto consenso entre el panel.

En general un elevado consenso que tan solo en la subpregunta de las concavidades subterráneas no se mostraron ni en acuerdo ni en desacuerdo.

\section{III.1.2. Factores socioeconómicos y medioambientales}

Los entrevistados aportaron bastante unanimidad en las respuestas respecto a si el turismo representaba generación de rentas complementarias, la mejora de la sostenibilidad y en la 
dinamización, o en la creación de empleo donde la puntuación media superó el 4, es decir que estaban bastante de acuerdo. No obstante, hay subpreguntas como en las que se aborda si la declaración de los PN del Interior-Norte de la Comunitat Valenciana, ha contribuido al desarrollo socioeconómico de los parques mejorando sus condiciones socioeconómicas o su contribución a preservar los modos de vida tradicionales donde la puntuación media sugiere el 3 (ni en acuerdo ni en desacuerdo).

Por lo que respecta a los métodos y la gestión innovadora, hay unanimidad en que o no existen o en su caso no han logrado resultados positivos. En cuanto a la contribución del turismo a la recuperación demográfica tan importante para reducir los desequilibrios territoriales y mantener las formas de vida tradicionales y su cultura es bastante negativa.

En definitiva, en los subapartados de esta pregunta, los resultados nos dejan muchas dudas sobre el haber alcanzado, sobre todo, los objetivos socioeconómicos, tanto desde la perspectiva del mantenimiento de la población como desde la perspectiva territorial y económica. Concretamente la tipología turística que tantas expectativas de desarrollo había generado con la declaración de estos espacios no se ha cumplido.

\section{III.1.3. La oferta turística}

En esta pregunta, el panel de expertos valoró positivamente las subpreguntas relacionadas con el alojamiento en viviendas particulares sobre el alojamiento comercial, aunque este (hoteles, hostales, casas rurales, entre otros) es valorado positivamente en cuanto a su calidad 4, (bastante de acuerdo). Estas se complementan también con un 4 en la mediana con la acogida positiva de los turistas en los núcleos de población de los PN, por lo tanto que la predisposición de la población es buena en general.

No obstante, existe un claro déficit en la gestión profesional de la oferta ante las exigencias de la demanda y carencias de la oferta comercial predominando lo cuantitativo sobre lo cualitativo como lo demuestra el valor intermedio presentado 3 (ni de acuerdo, ni en desacuerdo) lo que manifiesta una cierta indiferencia.

También se da una cierta indiferencia en el resultado en lo que respecta a la gestión, la información que se ofrece en los PP, o en el apoyo del sector público en la oferta. Ello tiene también su repercusión negativa en la oferta de actividades de ocio y recreación que es a la vez insuficiente, como lo son la oferta de turismo de naturaleza, aventura y deportivo, con valor intermedio 3. Estas valoraciones intermedias se completan con la de la accesibilidad con vehículo propio, si bien hay diferencias entre los diferentes PN del interior por las diferentes orografías que presentan estos espacios naturales entre sí.

Por último, las subpreguntas referentes a la oferta de la artesanía, la señalización de los recursos turísticos, aspectos básicos en la puesta en valor del recurso, y los puntos de información en estos espacios recibieron resultados negativos 2 pero por unanimidad.

Como conclusión, podemos deducir que la oferta en los PN presenta déficits importantes en casi todos los aspectos a pesar de la buena predisposición que presenta la población local para su desarrollo. 


\section{III.1.4. La demanda turística}

En esta pregunta el panel de expertos manifiesta una valoración neutra o negativa, lo que supone, a la vez, que el producto en los PN del interior-norte la Comunitat Valenciana no es bien valorado por la demanda. Destaca el alto grado de consenso en la subpregunta mejor valorada referente a las actitudes de mejora de los visitantes en los PN como lo demuestra el 0,5 de la desviación típica. También existe un importante grado de consenso en las respuestas a las subpreguntas referentes a la buena imagen y la adecuación de los atractivos turísticos a los visitantes o a la buena imagen que los factores que remodelan la oferta (limpieza, servicios, infraestructuras, entre otros) presentan. Muy significativas las respuestas negativas por consenso en cuanto al grado de conocimiento de la demanda ya no solamente de los mismos parques sino de sus productos, alojamientos comerciales, alojamientos no comerciales, lo que se refleja también de forma negativa en la escasez de acciones para incrementar la afluencia de visitantes.

$\mathrm{Al}$ respecto, entre las preguntas añadidas y contestadas por los panelistas como si ha mejorado la oferta multilingüe de servicios en los PN, si se ha incrementado el número de visitantes en los PN de origen provincial y autonómico, o si existe una verdadera oferta ecoturística de servicios que satisface las exigencias de gestión de los visitantes europeos más proclives a este tipo de gestión en los PN, la media es negativa sobre todo con respecto a la última cuestión 2 (bastante en desacuerdo) presentando un gran consenso en las respuestas por parte de los participantes.

\section{III.1.5. Infraestructuras y servicios}

En este apartado cabe destacar el consenso total en la media sobre la calidad y el tratamiento de las aguas residuales. También destacar un tratamiento aceptable (media 4) en torno al sistema de recogida de residuos sólidos, tendido eléctrico y los servicios sanitarios. En ello, sin duda, ha influido la aplicación de la Agenda 21 Local en estos espacios. Sin embargo, la media queda por debajo de 4 , entre 3,8 y 3,7 en la conectividad interna a los núcleos de población y sobre todo a los recursos turísticos, los servicios asistenciales, la conservación de la red de vías pecuarias, caminos y senderos en donde se evidencia la incidencia negativa de los recortes de estos años de crisis.

Por último, la aplicación de las nuevas tecnologías y el acceso a las redes telemáticas de alta capacidad (Internet) es la media peor valorada con 3,7 lo que se convierte dada su importancia para el desarrollo turístico como una asignatura pendiente.

\section{III.1.6. Financiación y gestión}

Las respuestas del panel de expertos han sido muy críticas y unánimes con la financiación (media 4,5 y desviación típica 0,6) y sobre todo con la gestión pública de estos espacios naturales. Así, tan solo de unos parámetros muy positivos entre bastante de acuerdo y totalmente de acuerdo la aseveración "de que los PN del espacio de estudio deben desarrollar mecanismos de financiación innovadores orientados a captar los recursos necesarios para su dinamización turística". 
También se ha registrado por unanimidad (desviación típica 0,7 ) en los resultados negativos de los panelistas en las subpreguntas como: "se destinan suficientes recursos financieros para dotar de recursos humanos a los PN".

Desde el punto de vista político se valora muy negativamente por consenso las acciones de la administración central de la Comunidad Autónoma y la Diputación en temas como el facilitar el desarrollo de la oferta y promoción de productos en estos espacios, no alcanzando la media de 3 . A ello se añade cuando la inadecuada oferta formativa dirigida a los prestadores de servicios turísticos, sobre todo a la aplicación de las nuevas tecnologías aunque también los agentes turísticos no han prestado demasiado interés por asistir a los cursos de formación convocados.

A modo de síntesis, en el Tabla 3, reflejamos las subpreguntas que han tenido un mayor consenso en las respuestas del panel de expertos y que giran en torno a las incidencias de los recursos turísticos, de la oferta, percepción de la demanda, estado de las infraestructuras y servicios, y la valoración de la financiación y gestión pública.

Tabla 3

SUBPREGUNTAS ANÁLISIS DE PARTIDA CON MAYOR NIVEL DE CONSENSO

\begin{tabular}{|c|c|c|}
\hline $\mathrm{N}^{\mathrm{o}}$ & Pregunta & $\begin{array}{l}\text { Desviación } \\
\text { típica }\end{array}$ \\
\hline \multicolumn{3}{|c|}{ INCIDENCIA DE LOS RECURSOS TURÍSTICOS } \\
\hline P 1.11 & $\begin{array}{l}\text { Que los senderos, caminos y pistas forestales de los PN del interior-norte de } \\
\text { la Comunitat Valenciana son fundamentales para el desarrollo de la actividad } \\
\text { turística. }\end{array}$ & 0.3 \\
\hline P.1.5 & $\begin{array}{l}\text { Los recursos paisajístico y de observación son básicos para el desarrollo } \\
\text { turístico de los PN del norte-interior de la Comunitat Valenciana }\end{array}$ & 0.4 \\
\hline P.1.6 & $\begin{array}{l}\text { El patrimonio histórico-monumental y etnológico es fundamental para el } \\
\text { desarrollo turístico de los PN del interior-norte de la Comunitat Valenciana }\end{array}$ & 0.7 \\
\hline P.1.7 & $\begin{array}{l}\text { El paisaje tradicional de los núcleos de población del interior-norte de los PN es } \\
\text { fundamental para el desarrollo turístico de la zona }\end{array}$ & 0.7 \\
\hline P.1.4 & $\begin{array}{l}\text { La flora y la fauna, son un recurso básico para el desarrollo turístico de los PN } \\
\text { del interior-norte de la Comunitat Valenciana }\end{array}$ & 0.7 \\
\hline \multicolumn{3}{|c|}{ INCIDENCIA DE LA OFERTA } \\
\hline P.3.7 & $\begin{array}{l}\text { Que la oferta gastronómica en los PN del interior-norte de la Comunitat } \\
\text { Valenciana es suficiente para la demanda existente }\end{array}$ & 0.7 \\
\hline \multicolumn{3}{|c|}{ PERCEPCIÓN DE LA DEMANDA } \\
\hline P.4.10 & Que las actitudes de los visitantes en los PN, han mejorado & 0.5 \\
\hline P.4.12 & $\begin{array}{l}\text { Que los PN han incrementado su número de visitantes de origen provincial y } \\
\text { autonómico }\end{array}$ & 0.7 \\
\hline \multicolumn{3}{|c|}{ INCIDENCIA DE LAS INFRAESTRUCTURAS Y SERVICIOS } \\
\hline P.5.4 & Infraestructuras de calidad de las aguas y la red de abastecimiento & 0.6 \\
\hline \multicolumn{3}{|c|}{ VALORACIÓN DE LA FINANCIACIÓN Y GESTIÓN PÚBLICA } \\
\hline P.6.5 & $\begin{array}{l}\text { Que se deben desarrollar mecanismos de financiación innovadores orientados a } \\
\text { captar los recursos necesarios }\end{array}$ & 0.6 \\
\hline
\end{tabular}

Fuente: elaboración propia. 


\section{III.2. Análisis prospectivo}

Una vez realizada la fase de análisis de partida, esta investigación abordó la fase de prospectiva (Bloque II. Tabla 2) con el objetivo de alcanzar los principales factores que podrían limitar el futuro o activar el desarrollo sostenible turístico de los PN del interior-norte de la Comunitat Valenciana.

\section{III.2.1. Factores que pueden representar un freno a la evolución turística}

Entre los factores bastante limitantes con valoraciones de 4, se encuentran, según los resultados (Tabla 4) la escasa o mala inversión pública, la pérdida de actividades tradicionales, la falta de ordenación y planificación, la falta de relación entre los agentes públicos y privados, el envejecimiento de la población y el déficit de preparación de los recursos humanos. También se presentan como negativos (cerca de la media 4) la falta de alojamiento comercial, de oferta complementaria, de servicios comerciales, de infraestructuras, el impacto medioambiental y paisajístico, el bajo nivel competitivo de los parques, la motivación de la población local y el déficit en los servicios básicos. En todas estas subpreguntas, en general, existe bastante unanimidad en las respuestas visto los resultados de la desviación típica, presentándose menos consenso en el impacto medioambiental y paisajístico, en el déficit de los servicios básicos y la motivación de la población local.

Igualmente hay unanimidad en determinar como factores limitantes la escasez de agentes privados y su organización en redes, y sobre todo la ausencia de marca-destino y la existencia de núcleos de población de escasa entidad.

Tabla 4

FACTORES QUE PUEDEN REPRESENTAR UN FRENO A LA EVOLUCIÓN TURÍSTICA

\begin{tabular}{|l|l|c|}
\hline $\mathrm{N}^{\circ}$ & Pregunta & Desviación típica \\
\hline \multicolumn{2}{|l|}{ FACTORES QUE PUEDEN REPRESENTAR UN FRENO A LA EVOLUCIÓN TURÍSTICA } \\
\hline P 7.16 & Escasa o nula inversión pública & 0.7 \\
\hline P.7.3 & Pérdida de actividades tradicionales & 0.7 \\
\hline P.7.15 & Falta de relación entre los agentes públicos y privados & 0.7 \\
\hline P.7.11 & Fuerte estacionalidad & 0.7 \\
\hline P.7.10 & Escasez de servicios comerciales & 0.7 \\
\hline
\end{tabular}

Fuente: elaboración propia.

\section{III.2.2. Acciones para activar el desarrollo turístico en los parques naturales}

Es una de las preguntas donde se ha producido mayor consenso entre los panelistas, como se puede apreciar en las bajas desviaciones típicas. En esta pregunta se dan hasta 13 subpreguntas, donde los valores oscilan entre el 4 y 5 (casi el totalmente de acuerdo con la acción propuesta) como lo refleja la desviación típica (Tabla 5). Por lo tanto hay bastante de acuerdo 
en las acciones propuestas para activar el dinamismo turístico en los PN en torno a la reconversión de recursos en productos e invertir en los existentes, mejorar las infraestructuras de comunicación y potenciar aspectos de la nueva gobernanza.

Tabla 5

ACCIONES PARA EL DESARROLLO TURÍSTICO

\begin{tabular}{|c|c|c|}
\hline $\mathrm{N}^{\mathrm{o}}$ & Pregunta & $\begin{array}{l}\text { Desviación } \\
\text { típica }\end{array}$ \\
\hline \multicolumn{3}{|c|}{ ACCIONES PARA EL DESARROLLO TURÍSTICO } \\
\hline P.8.5 & Potenciar los recursos gastronómicos & 0.6 \\
\hline P.8.4 & $\begin{array}{l}\text { Mayor inversión en la conservación de las redes de caminos y senderos } \\
\text { del interior-norte de la Comunitat Valenciana para un aprovechamiento } \\
\text { sostenible de los mismos }\end{array}$ & 0.6 \\
\hline P.8.2 & Más inversión para la adecuación de los recursos culturales y patrimoniales & 0.7 \\
\hline P.8.17 & Convertir recursos en productos estructurados & 0.7 \\
\hline P.8.21 & $\begin{array}{l}\text { Potenciar la nueva gobernanza con la creación de consorcios entre agentes } \\
\text { públicos y privados para impulsar la promoción y comercialización de } \\
\text { productos }\end{array}$ & 0.7 \\
\hline P.8.8 & Mejorar las infraestructuras de comunicación & 0.7 \\
\hline P.8.1 & $\begin{array}{l}\text { Más inversión en la conservación de los recursos naturales para una mayor } \\
\text { sostenibilidad de los recursos }\end{array}$ & 0.7 \\
\hline
\end{tabular}

Fuente: elaboración propia

También aparecen con valores próximos al 4 (3.9) (bastante de acuerdo) subpreguntas como, reforzar la calidad del alojamiento comercial en la dotación de servicios complementarios; adecuar las infraestructuras básicas (agua, energía, entre otras.); adecuar la recogida y tratamiento de residuos sólidos; e invertir en oferta de ocio y recreación

Finalmente, también se alude a la creación de empresas de carácter receptivo, ofertar más servicios comerciales y en la creación de un centro de reservas propio en los PN. Por lo tanto las valoraciones de los entrevistados en las acciones propuestas han sido respondidas con bastante unanimidad y consenso. Es decir que a la vista de los resultados hay muchas acciones básicas pendientes de desarrollar en estos espacios naturales del interior-norte de la Comunitat Valenciana.

\section{III.2.3. El desarrollo turístico sostenible en los parques naturales}

Los resultados de esta pregunta también se ven acompañados de un gran consenso en las subpreguntas propuestas como se puede deducir de la desviación típica sobre todo en aquellas preguntas mejor valoradas, destacando con un consenso cercano al $5(4,7)$ las referentes a la ordenación del territorio en los PN, en el marco de la legislación propia y del consenso de todos los agentes implicados; al desarrollo turístico en los PN con carácter integral (estructura socioeconómica y medioambiental); y a los alojamientos y servicios complementarios que deben salvaguardar la autenticidad de sus espacios (Tabla 6). 
Tabla 6

PRIORIDADES PARA EL DESARROLLO TURÍSTICO SOSTENIBLE

\begin{tabular}{|l|l|c|}
\hline$N^{\circ}$ & Pregunta & $\begin{array}{c}\text { Desviación } \\
\text { típica }\end{array}$ \\
\hline \multicolumn{2}{|l|}{ PRIORIDADES PARA EL DESARROLLO TURISICO SOSTENIBLE } \\
\hline P.9.5 & $\begin{array}{l}\text { Que el desarrollo turístico debe ser integrado en la estructura socio- } \\
\text { económica y medioambiental }\end{array}$ & 0.5 \\
\hline P.9.6 & $\begin{array}{l}\text { Los alojamientos y sus servicios complementarios deben salvaguardar la } \\
\text { autenticidad de sus espacios }\end{array}$ & 0.6 \\
\hline P.9.1 & $\begin{array}{l}\text { La ordenación del territorio entre PN, en el marco de la legislación propia } \\
\text { debe ser fruto del consenso en todo lo implicado }\end{array}$ & 0.7 \\
\hline P.9.7 & Aplicar medidas de capacidad de acogida y de impacto & 0.7 \\
\hline
\end{tabular}

Fuente: elaboración propia.

A estas subpreguntas les siguen otros tres planteamientos que están en la media 4 o superior (bastante de acuerdo), y que también presentan unanimidad en los resultados como son la relevancia de la aplicación de la capacidad de acogida; la importancia de que se generen productos bien estructurados; y la importancia de reducir la estacionalidad.

\section{CONCLUSIONES}

Los parques naturales del interior-norte de la Comunitat Valenciana, objeto de esta investigación, a pesar de su riqueza natural y paisajística, reflejada en la potencialidad de sus recursos, presentan un gran déficit en su puesta en valor y transformación en productos.

Por otra parte, la oferta turística en los parques naturales del interior-norte de la Comunitat Valenciana, esta se presenta muy limitada tanto en el alojamiento, como en los servicios y el ocio. Las empresas presentes en los municipios de estos parques, aunque escasas, son básicamente empresas de alojamiento y restauración. La crisis económico-financiera actual ha afectado seriamente al sector, lo que se refleja en la desaparición de alojamientos turísticos y restaurantes o en la bajada de su categoría. En el caso de las empresas especializadas en turismo activo se reducen a una en el parque natural de la Sierra de Espadán, otra en el parque natural del Penyagolosa y dos en el parque natural de la Tinença de Benifassà, una oferta que no satisface una demanda creciente. No obstante, se observa una creciente oferta de rutas y senderos aunque con déficits en su activación como productos.

La demanda dentro de estos parques es creciente aunque sufre de estacionalidad, la afluencia de los visitantes en el parque natural de la Sierra de Espadán se concentra sobre todo en los meses de mayo, abril y noviembre, en el parque natural del Penyagolosa es el mes de agosto el que tiene más visitantes y el parque natural de la Tinença de Benifassà la mayoría de las visitas no concertadas se registran en el mes de mayo y las concertadas en el mes de noviembre. No obstante, la demanda desconoce no solamente los mismos parques sino también sus productos, alojamientos comerciales, alojamientos no comerciales, entre otros, lo que refleja la escasez de acciones realizadas para subsanar este desconocimiento e incrementar la demanda. 
Respecto a las infraestructuras y servicios son positivas las acciones desarrolladas tanto de carácter general como básicas (abastecimiento de aguas y su tratamiento, por ejemplo). Sin embargo, la conectividad interna entre sus núcleos de población es deficiente, al igual que la conservación de las vías pecuarias, caminos y senderos. Muy deficientes se presentan para los panelistas las redes telemáticas de alta capacidad.

En cuanto al grado de financiación y gestión se deben desarrollar, según las entrevistas, mecanismos de financiación innovadores orientados a captar los recursos necesarios para la dinamización turística y para ello es conveniente aplicar los enfoques de la nueva gobernanza con la creación de consensos entre los agentes públicos y privados.

Igualmente, el desarrollo turístico de los parques naturales del interior-norte de la Comunitat Valenciana no ha sido satisfactorio en la consecución de los objetivos territoriales y socioeconómicos como de la perspectiva del mantenimiento de la población.

Finalmente, es necesario potenciar el desarrollo de los parques naturales del interior-norte de la Comunitat Valenciana con una serie de estrategias y programas que potencian los valores ecológicos, productivos y sociales. Ello supone reforzar tres funciones fundamentales como la conservación del patrimonio natural y cultural; el desarrollo socioeconómico integral; y potenciar el uso público y social de estos espacios naturales incluyendo lógicamente las actividades de turismo, ocio y recreación.

\section{BIBLIOGRAFÍA}

ANTON, S., BLAY, J. y SALVAT, J. (2008): “Turismo, actividades recreativas y uso público en los parques naturales. Propuesta para la conservación de los valores ambientales y el desarrollo productivo local" en Boletín de la Asociación de Geógrafos Españoles n 48, 5-38.

BUCKLEY, R. (2003): "Research Note Environmental Inputs and Outs puts in Ecotourism: Geotourism with a Positive Triple Bottom Line?". Journal of Ecotourism, Vol. 2, n 1, 76-82.

CAPDEPÓN, M. (2013): El papel de los parques naturales como elementos de diversificación en el marco de la renovación de los destinos turísticos consolidados. Tesis Doctoral. Universidad de Alicante. Instituto Universitario de Investigaciones Turísticas. Disponible en: https://rua.ua.es/dspace/bitstream/10045/36498/1/tesis_capdepon_frias.pdf

CAPDEPÓN, M. (2015): "El papel de los parques naturales en los procesos de diversificación turística: una aplicación al litoral alicantino". Cuadernos de Turismo no 35, 43-69.

CELAYA, A.M. (2005): Un proceso estratégico para el turismo de Córdoba. Tesis Doctoral defendida el 5 de octubre de 2005. ETEA. Facultad de Ciencias Económicas y Empresariales. Universidad de Córdoba.

EPLER, M. (2002): Ecotourism: principles, practices \& policies for sustainability. Paris. United Nations Environment Programme.

FENNELL, D.A. y DOWLING, R.R. (2003): Ecotourism Policy and Planning, CABI Publishing, Wallingford.

FLORES, D. (2008): Competitividad sostenible de los espacios naturales protegidos como destinos turísticos. Un análisis comparativo de los parques naturales Sierra de Aracena y Picos de Aroche y Sierras de Cazorla, Segura y las Villas. Universidad de Huelva. Disponible en http://www.eumed.net/tesis-doctorales/\#2008 
GARROD, B., y FYALL, A. (2005): "Revisting Delphi: The Delphi Technique in tourism research", en Tourism Research Methods (Ritche, B., Burns,P. y Palmer, C. Eds.), CAB International.

GREEN, H., HUNTER, C. y MOORE, B. (1990): “Assessing the environmental impact of tourism development: use of the Delphi technique". Tourism Management $\mathrm{n}^{\circ} 11$ (2) 111120.

HSU, C y SANDFORD, B (2007): “The Delphi Technique: Making Sense of Consensus”. Practical Assessment, Research \& Evaluation, Vol. 12, nº 10.

HUÉSCAR, A. (2007): "La situación actual del turismo internacional”. En: Turismo en los espacios litorales. (López Olivares, D., Ed.) Valencia. Tirant lo Blanch. 15-35

KAYNAK, E., BLOOM, J. y LEIBOLD, M. (1994): "Using the Delphi technique to predict future tourism potential". Market Intelligence and Planning $\mathrm{n}^{\circ} 7$ (12) 18-29.

LANDETA, R.J. (1999): El método Delphi: Una Técnica o Precisión para la incertidumbre. Barcelona. Ariel.

LINSTONE, H.A y TUROFF, M. (1975): The Delphi Method. Techniques and applications. Addison-Wesley.

LINSTONE, H.A y TUROFF, M. (2002): The Delphi Method: Techniques and applications. Disponible en http://citeseerx.ist.psu.edu/viewdoc/download?rep=rep1\&type=pdf $\&$ doi=10.1.1.224.1531 Consultado 13 de febrero de 2017.

LÓPEZ, D. (2005): "Reflexiones sobre el desarrollo rural en la Unión Europea y la Comunidad Valenciana". Quaderns Agroambientals. Institut Valencià d'Investigació i Formació Agroambiental (IVIFA). Valencia.

LÓPEZ D. et al. (2005): "Cambios y transformaciones en el actual modelo turístico de España”. Annals of Tourism Research en Español n 7 (2) 423-446.

LÓPEZ D. y PULIDO, J.I. (2007): "El estado de la cuestión” En: La Actividad Turística Española. AECIT. Madrid. Edit. Ramón Areces.

LÓPEZ, D. (Ed.) (2010): Turismo y gestión de espacios protegidos. Valencia. Tirant lo Blanch.

LÓPEZ, D. (2014): Los recursos turísticos. Evaluación, ordenación y planificación turística. Estudio de casos. Valencia. Tirant lo Blanch.

LÓPEZ, F. (2001): Factores condicionantes de la ventaja competitiva y de los resultados de las agencias de viaje en España: un estudio empírico de sus aspectos estratégicos más relevantes. Tesis Doctoral. Departamento de Economía de la Empresa, Universidad Autónoma de Barcelona.

LUZARDO, M.A. (2014): Potencialidades y estrategias para la puesta en valor turístico del frente de agua y casco histórico de los puertos de Alta Gracia (Venezuela). Facultad de Ciencias Económicas y Empresariales. Departamento de economía aplicada. Universidad de Málaga.

MARCHENA, M. et al (1992): Ocio y turismo en los parques naturales andaluces. Sevilla. Dirección General de Turismo.

MOUTINHO, L. y WITT, S.F. (1995): "Forecasting the tourism environment using a consensus approach". Journal of Travel Research $\mathrm{n}^{\circ} 33$ (4), 46-50. 
MUÑOZ, J.C. (2008): "El turismo en los espacios naturales protegidos españoles algo más que una moda reciente". Boletín de la Asociación de Geógrafos Españoles, nº 46, 291303.

NEWSONE, D., DOWLING, R. R. y MOORE, S.A. (2005): Wildlife Tourism, Channel View Publications. Clevedon.

NORTHCOYE, J., LEE, D., CHOK, S. y WEGNER, A. (2008): “An Email-Based Delphi. Approach to tourism program evaluation: Current stakeholders in research design" en Current Issues in Tourism $\mathrm{n}^{\circ} 11$ (3) 296-297.

PULIDO, J.I. (2005): Criterios para una política turística sostenible en los parques naturales de Andalucía. Junta de Andalucía, Consejería de Turismo, Comercio y Deporte. Sevilla.

PULIDO, J.I. (2007): “El turismo en el desarrollo económico de los parques naturales andaluces. Un análisis Delphi de los principales déficits". Revista de Estudios Empresariales, $2^{\mathrm{a}}$ época, $\mathrm{n}^{\circ} 1,100-137$.

PULIDO, M. y PULIDO J.I. (2016): “¿Existe un buen modelo para implementar la gobernanza en destinos turísticos? La opinión de los expertos en sostenibilidad en los modelos actuales de gestión turística", en Sostenibilidad en los modelos actuales de gestión turística (López Olivares, D., Edit). Valencia. Tirant lo Blanch, 57-85.

SANCHO. A (Dir.) (2003): Apuntes de metodología de la investigación en turismo. Organización mundial del turismo.

SOLSONA, J. (2010): Desarrollo turístico en Espacios Rurales, Análisis de la situación prospectiva. Estudio Aplicado a la Comunitat Valenciana. Tesis doctoral. Universitat Jaume I, Departamento de Historia, Geografía y Arte.

SOLSONA, J. y LÓPEZ, D. (2012): "Factores de localización y desarrollo turístico en el espacio rural de la Comunitat Valenciana" en Boletín de la Asociación de Geógrafos Españoles, $\mathrm{n}^{\circ}$ 59, 221-243.

VERA, J.F. (Coord.) (1997): Análisis territorial del turismo. Barcelona. Ariel Geografía.

VERA, J.F. (Coord.) (2011): Análisis territorial del turismo y planificación de destinos turísticos. Valencia. Tirant lo Blanch.

WEAVER, D.B. (2001): “Ecotourism as mass tourism: contradiction or reality?". The Cornell Hotel and Restaurant Administration Quarterly $\mathrm{n}^{\circ}$ 42(2), 104-112

WEBER, K y LADKIN, A (2003): "The Convention Industry in Australia and the United Kingdom; key Issues and competitive Forces". Journal of Travel Research, $\mathrm{n}^{\circ} 42$ (2), $125-132$.

\section{PÁGINAS WEB}

AGÈNCIA VALENCIANA DEL TURISME (AVT):

http://www.turisme.gva.es/opencms/opencms/turisme/es/contents/estadistiquesdeturisme/ anuario/oferta_turistica/oferta_turistica_municipal_comarcal.html

EUROPARC España (2009):

http://www.redeuroparc.org/img/publicaciones/memoria2009.pdf

INSTITUTO NACIONAL DE ESTADÍSTICA (INE):

http://www.ine.es/jaxi/menu.do?type=pcaxis \&path=\%2Ft11\%2Fe162eotr\&file=inebase 
INSTITUTO DE ESTUDIOS TURÍSTICOS (IET):

http://estadisticas .tourspain .es/es-ES/turismobase/Paginas/default.aspx

MINISTERIO DE AGRICULTURA, ALIMENTACIÓN Y MEDIO AMBIENTE:

http://www.magrama.gob.es/es/biodiversidad/temas/espacios-protegidos/red-natura-2000/ ORGANIZACIÓN MUNDIAL DEL TURISMO (OMT):

http://www2.unwto.org/es 